\title{
Supervised Component Analysis for EELS Mapping
}

\author{
Shixin Wang
}

Micron Technology, Inc., Boise, ID, USA

Many multivariate statistical analysis methods have been used to analyze EELS and EDS spectrum images. They include principal component analysis (PCA) [1, 2], non-negative matrix factorization (NMF) [3], independent component analysis (ICA) [4], and multivariate curve resolution (MCR) [5]. The primary goal of all those methods is to reduce dimensionality of the dataset by identifying a few major components so that the whole dataset can be approximated by the linear combination of the components.

In theory, PCA gives the best component representation in the sense of least square. However, because it involves rotating vector space bases, it is often difficult to interpret the physical meaning of the analysis results. For multivariate dataset such as EELS mapping and EDS mapping, NMF and ICA is usually the preferred method so that the physical meaning is preserved during the component analysis. With priori knowledge of the specimen, we may employ a straight forward component analysis on user identified components.

The component analysis assumes the dataset can be approximated by a linear combination of a few components. This discussion is based on EELS mapping, but the method can be applied to other spectral imaging methods. With EELS mapping, we obtain a 3D spectrum image (SI), with $n$ and $m$ for spatial dimensions and a $3^{\text {rd }}$ dimension for energy loss spectra. We represent an $n$ by $m$ EELS SI data with energy channels $k$ by matrix $\boldsymbol{B}$, which has dimension of $k$ by $n^{*} m$. We define a component matrix $\boldsymbol{A}$ with dimension of $k$ by $p$, where $p$ is the number of components. We also define a coefficient matrix $\boldsymbol{X}$ with dimension of $p$ by $n^{*} m$. We assume,

$$
B=A X+E,
$$

where $\boldsymbol{E}$ is the residue matrix and is to be minimized in the sense of least square. When $\boldsymbol{E}$ is minimized, we say $\boldsymbol{A} \boldsymbol{X}$ gives the best estimate of $\boldsymbol{B}$ for the given components. The matrix $\boldsymbol{X}$ gives the spatial map of each component contribution.

A computer program is built with Gatan ${ }^{\circledR}$ DigitalMicrograph $($ script to carry out component selection, data pre-processing, and component analysis (Fig. 1A). A good understanding of the specimen can be obtained through prior knowledge of the sample and through careful study of EELS spectra in the dataset. Averaged spectrum can be selected as the individual component by interactively picking out a summed spectra from an area of interest on the SI.

The matrix $\boldsymbol{X}$ is solved with matrix solution of least square problem via QR decomposition [6]. A set of resulting component maps are given in Fig. 1. There we notice the successful separation of W, $\mathrm{Si}$ and $\mathrm{SiO}_{2}$ phases. The signals over that energy range are heavily overlapped and they are difficult to be separated in regular window-based mapping method. The residue map is also shown, from which we can study the error and further optimize the component analysis.

The supervised component analysis is not limited to selecting a few sample spectra from the dataset. It can be extended to use a selected spectra from a standard library. If we pick out components from the SI, we may clean up the dataset with background subtraction and balance signal strength with intensity normalization. For multiple-element SI, step-wise background subtraction for different elements is necessary. This operation is splitting matrix $\boldsymbol{B}$ to $\boldsymbol{B}^{\boldsymbol{s}}$, for signal, and $\boldsymbol{B}^{\boldsymbol{b}}$, for background. We select to discard $\boldsymbol{B}^{\boldsymbol{b}}$, because it does not contain 
elemental information. Step-wise normalization is valid in terms of keeping physical interpretation if we are picking out components from the processed SI, but is generally not valid if we use standard EELS edge library. This step-wise operation is demonstrated in Fig. 2, where the component mapping is calculated using the whole energy range. Step-wise calculation with a subset of the energy range might be used to show featured phases with better definition.

\section{References:}

[1] Trebbia, P. and Bonnet, N. Ultramicroscopy 34 (1990), p. 165.

[2] Lucas, G. et al, Micron 52-53 (2013), p. 49.

[3] Lee, D. D. and Seung, H. S. Nature 401 (1999), p. 788.

[4] de la Pe na, F. et al, Ultramicroscopy 111 (2011), p. 169.

[5] Muto, S., Yoshida, T., and Tatsumi, K. Materials Transactions 50 (2009), p. 964.

[6] Watkins, D. S., "Fundamentals of Matrix Computations", $3^{\text {rd }}$ Ed., John Wiley \& Sons, Hoboken.

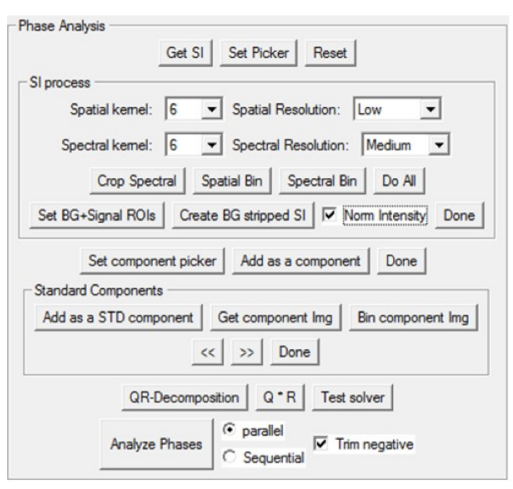

(A)

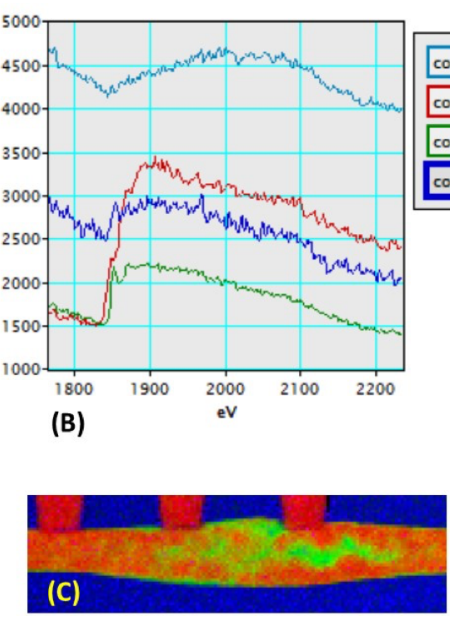

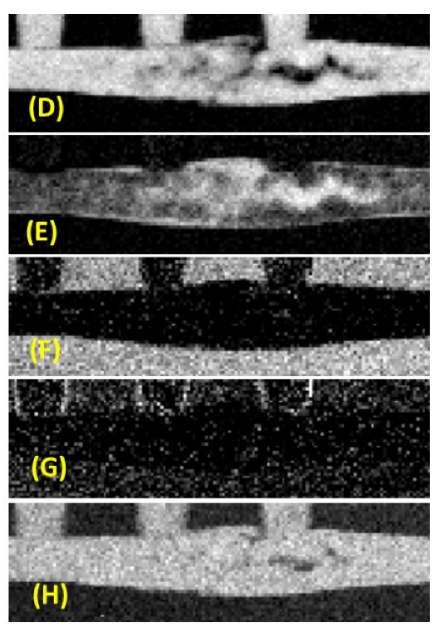

(H)

Figure 1. (A) The software interface. (B) The selected four components. (C) Color overlay of first three components distributions. (D) Component-1 map corresponding to $\mathrm{W}$ distribution. (E) Component-2 map corresponding to Si distribution. (F) Component-3 map corresponding to $\mathrm{SiO}_{2}$ distribution. (G) Component-4 map corresponding to TiN distribution. (H) Residue map.

(A)

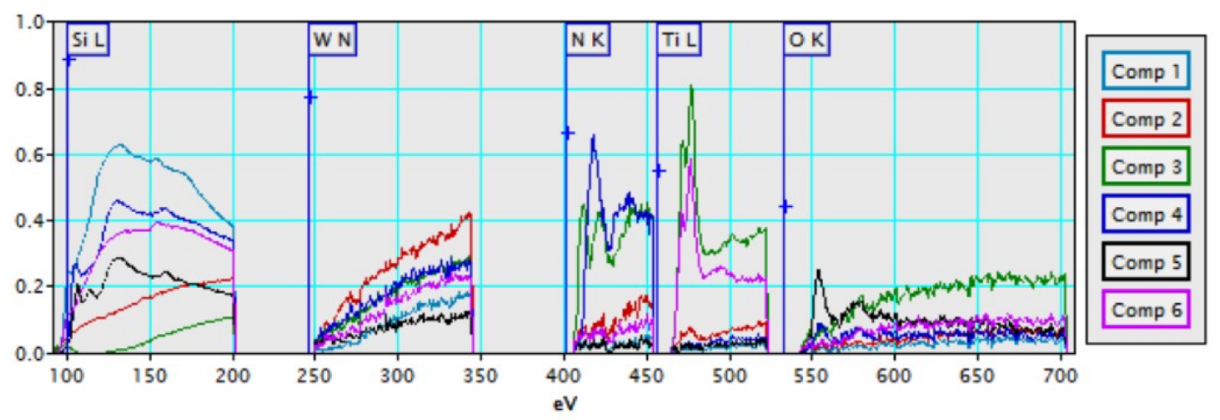

(B)
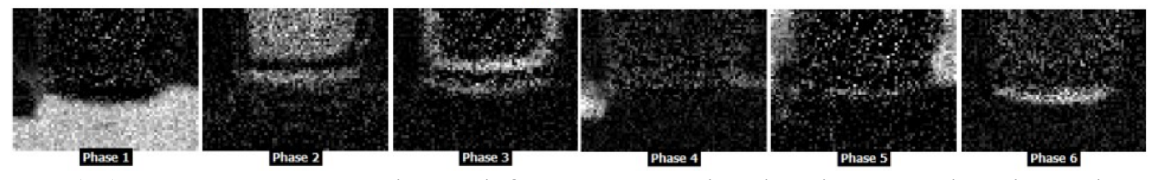

Figure 2. (A) Components selected from step-wise background stripped and normalized SI.

(B) Maps of the components using the whole energy range. 